\title{
The effects of iloprost on lung injury induced by skeletal muscle ischemia-reperfusion
}

\author{
Erer $\mathrm{D}^{1}$, Dursun $\mathrm{AD}^{2}$, Oktar $\mathrm{GL}^{1}$, Iriz $\mathrm{E}^{1}$, Zor $\mathrm{MH}^{1}$, Elmas $\mathrm{C}^{3}$, Donmez $\mathrm{T}^{1}$, Kirisci $\mathrm{M}^{1}$, \\ Comu FM로 Arslan $\mathrm{M}^{4}$ \\ Department of Cardiovascular Surgery, Faculty of Medicine, Gazi University, Ankara, Turkey. \\ marslan36@yahoo.com
}

\begin{abstract}
Purpose: The aim of this study was to investigate the effects of iloprost (I) on lung injury as a remote organ following skeletal muscle ischemia-reperfusion injury in a rat model.

Materials and methods: Twenty-four Wistar Albino rats were randomized into four groups $(n=6)$. Laparotomy was performed in all groups under general anesthesia. Only laparotomy was applied in Group S (Sham). Ischemia reperfusion group (Group I/R) underwent ischemia and reperfusion performed by clamping and declamping of the infrarenal abdominal aorta for 120 minutes. Group iloprost (Group I) received intravenous infusion of iloprost $0.5 \mathrm{ng} / \mathrm{kg} / \mathrm{min}$, without ischemia and reperfusion. Group I/R/I received intravenous infusion of iloprost $0.5 \mathrm{ng} / \mathrm{kg} / \mathrm{min}$ immediately after 2 hours of ischemia. At the end of the study, lung tissue was obtained for determining total oxidant status (TOS) and total antioxidant status (TAS) levels, histochemical and immunohistochemical determination.

Results: Diffuse lymphocyte infiltration was detected in immunohistochemical examination of lung tissue in Group $\mathrm{I} / \mathrm{R}$. The connective tissue around bronchi, bronchioles and vessel walls was found to be increased. Although minimal local lymphocyte infiltration was detected in some fields in Group I/R/I, the overall tissue was found to be similar to Group S. iNOS expression was significantly higher in Group I/R, when compared with Group $S$ and significantly lower in Group I/R/I compared to Group I/R.

TOS levels were significantly higher in Group I/R, when compared with groups $S$ and I $(p=0.028, p=0.016$, respectively) and significantly lower in group I/R/I, when compared with Group I/R $(p=0.048)$. TAS levels were significantly higher in Group I/R, when compared with groups $S, I(p=0.014, p=0.027$, respectively) and significantly lower in Group I/R/I, when compared with Group I/R ( $p=0.032)$.

Conclusion: These results indicate that administration of iloprost may have protective effects against ischemia reperfusion injury (Fig. 8, Tab. 1, Ref. 30). Text in PDF www.elis.sk.

Key words: ischemia-reperfusion, total oxidant status, total antioxidant status, iloprost, iNOS, lung tissue, rat.
\end{abstract}

Ischemia/reperfusion (I/R) induced tissue injury is one of the most important problems in a number of surgical procedures including extremity revascularization, abdominal aortic surgery, replantation, transplantation and muscular flap reconstruction (1, 2 ). Reperfusion of ischemic tissues results in both a local and a systemic inflammatory response that, in turn, may result in widespread microvascular dysfunction and altered tissue barrier function (3). Skeletal muscle I/R elicits oxidative stress and causes inflammation in lung tissue that may lead to lung injury.

Iloprost (I), a stable prostacyclin-mimetic drug, is widely prescribed to treat vascular features of systemic sclerosis (Raynaud's

\footnotetext{
${ }^{1}$ Department of Cardiovascular Surgery, Faculty of Medicine, Gazi University, Ankara, Turkey, ${ }^{2}$ Department of Physiology, Faculty of Medicine, Kirikkale University, Kirikkale, Turkey, ${ }^{3}$ Department of Histology and Embryology, Faculty of Medicine, Gazi University, Ankara, Turkey, and ${ }^{4}$ Department of Anesthesiology, Faculty of Medicine, Gazi University, Ankara, Turkey

Address for correspondence: M. Arslan, Dr, Gazi University Medical Faculty, Department of Anesthesiology and Reanimation, 06510 Ankara, Turkey.

Phone: +90.312.2026739, Fax: +90.312.2024166
}

phenomenon, digital ulcers and pulmonary arterial hypertension) on the grounds of its well known vasodilatory and antiaggregant effects. Beside these actions, Iloprost appears to have also an antioxidative effect which substantially adds to the spectrum of his "nonconventional" (e.g. antifibrotic and angiogenetic) actions $(4,5)$.

The aim of the present study was to examine the potential protective effects of Iloprost on lung damage induced by skeletal muscle I/R in a rat model, using both biochemical and histopathological aspects.

\section{Materials and methods}

\section{Experimental groups}

Twenty-four adult Wistar-albino rats, weighing 200 to $250 \mathrm{~g}$ were used in this study. Rats were housed in cages at an average temperature of $22{ }^{\circ} \mathrm{C}$ in a light dark cycle-controlled environment with free access to food and tap water. The protocols of this experimental study were approved by the Animal Ethics Committee of Gazi University. All animals received human care in compliance with the "Principles of Laboratory Animal Care" formulated by the National Society for Medical Research and the "Guide for the 
Care and the Use of Laboratory Animals" prepared by the National Academy of Science and published by the National Institutes of Health (NIH publication No. 85-23, revised in 1985).

\section{Study design}

Rats were randomized into four groups $(n=6)$. The sham group (Group S) underwent midline laparotomy and dissection of the infrarenal abdominal aorta (IAA) without cross-clamping; ischemia reperfusion group (Group I/R) underwent laparotomy and cross-clamping of the IAA for 120 minutes and then 120 minutes of reperfusion; Group I underwent laparotomy and received intravenous infusion of Iloprost $(0.5 \mathrm{ng} / \mathrm{kg} / \mathrm{min})$, without ischemia and reperfusion; Group I/R/I received intravenous infusion of Iliprost $(0.5 \mathrm{ng} / \mathrm{kg} / \mathrm{min})$ immediately after 120 minutes of ischemia.

\section{Aortic occlusion and ischemia reperfusion}

Rats were anesthetized with ketamine hydrochloride (Ketalar, $50 \mathrm{mg} / \mathrm{kg}$, intramuscularly, Parke-Davis, Eczacibasi, Istanbul, Turkey) and xylazine hydrochloride (Alfazyne, 2\%, Ege Vet, Izmir, Turkey). Anesthesia was maintained by an additional muscular injection of ketamine hydrochloride and xylazine hydrochloride. The surgical procedures were performed while the rats were placed in a supine position under a heating lamp. The abdomen was shaved, the skin was prepared aseptically and a midline laparotomy was performed. The abdominal aorta was exposed and clamped using an atraumatic microvascular clamp. The aortic occlusion was confirmed by the loss of the distal arterial pulsation. The skin incision was closed and covered with a plastic wrap to maintain the body temperature and fluid balance. After 120 minutes of ischemia, the microvascular clamp was removed and lower extremities were reperfused for 120 minutes. At the end of the reperfusion period, all rats were sacrified under anesthesia and left lung were harvested for biochemical, histopatological and immunohistopatological analyses.

\section{Biochemical examination}

Blood samples were obtained after induction of anesthesia and at the end of the surgery. The samples were centrifuged at 3000 revolutions per minute for 10 minutes to separate plasma and then blood samples were stored at $-80{ }^{\circ} \mathrm{C}$ until analysis.

\section{Measurement of serum total oxidant status (TOS)}

Plasma total oxidant status (TOS) levels were determined using a commercially available kit, developed by Erel (6) (REL assay diagnostics, Mega Tip, Gaziantep, Turkey). In this method, the oxidants present in the sample oxidize the ferrous ion-o-dianisidine complex to ferric ion. The oxidation reaction is enhanced by glycerol molecules, which are abundantly present in the reaction medium. The ferric ion produces a colored complex with xylenol orange in an acidic medium. The color intensity, which can be measured spectrophotometrically, is related to the total amount of oxidant molecules present in the sample. The assay is calibrated with hydrogen peroxide, and the results are expressed as $\mu$ mol $\mathrm{H}_{2} \mathrm{O}_{2}$ equivalent/L. Hydrogen peroxide and other derivatives of peroxides, produced physiologically in organisms and occurring in higher concentrations under some pathologic conditions, diffuse into plasma. The level of total peroxide was measured and expressed as TOS in this study.

\section{Measurement of serum total antioxidant status (TAS)}

Plasma total antioxidant status (TAS) levels were determined using a commercially available kit developed by Erel (REL assay diagnostics, Mega Tip, Gaziantep, Turkey) (7). In this method, hydroxyl radical, which is the most potent radical, is produced via Fenton reaction. In the classical Fenton reaction, the hydroxyl radical is produced by mixing of ferrous ion solution and hydrogen peroxide solution. In the most recently developed assay by Erel, the same reaction is used. In the assay, ferrous ion solution, which is present in the Reagent 1 , is mixed with hydrogen peroxide, which is present in the Reagent 2 . The sequentially produced radicals such as brown-colored dianisidinyl radical cation, produced by the hydroxyl radical, are also potent radicals. In this assay, antioxidative effect of the sample against the potent free radical reactions, which is initiated by the produced hydroxyl radical, is measured. The assay has got excellent precision values, which are lower than $3 \%$. The results are expressed as mmol Trolox equivalent.

\section{Immunohistochemical method}

All tissues were transferred immediately in to $10 \%$ formal saline for 72 hours, dehydrated in graded alcohols and embedded in paraffin wax. $4 \mu \mathrm{m}$ sections were cut on a microtome (Leica SM 2000, Germany) and mounted on polylizine-coated sides (Menzel-Glaser, Braunschweig, Germany). Some part of tissue samples were stained with Masson-trichrome for the evaluation of ultrastructural changes and inducible nitric oxide synthase (iNOS) expression was evaluated in other part of the samples for immunohistochemical examination. After an overnight incubating at $37^{\circ} \mathrm{C}$ and then for $1 \mathrm{~h}$ at $60{ }^{\circ} \mathrm{C}$, slides were de-waxed by two chances of xylene (10 minutes each). The slides were then placed in 100\%, 96 $\%$ and $80 \%$ ethanol for 10 minutes each, followed by two 5 minutes changes of distilled water. Then tissues were circumscribed with a pap pen (Super PAP Pen, PN IM3580, Becman Coulter Company, France). After washing with distilled water and with phosphate buffer saline (PBS, $\mathrm{pH}=7.4$ ), endogenous enzymeswere blocked using hydrogen peroxide in 10 minutes. Following a PBS wash, slides were blocked using ready to use Ultra block (Cat: 859043,

Tab. 1. TOS and TAS parameters of the study groups (mean \pm SD).

\begin{tabular}{lcccc}
\hline & $\begin{array}{c}\text { Group s } \\
(\mathrm{n}=6)\end{array}$ & $\begin{array}{c}\text { Group I } \\
(\mathrm{n}=6)\end{array}$ & $\begin{array}{c}\text { Group I/R } \\
(\mathrm{n}=6)\end{array}$ & $\begin{array}{c}\text { Prup I/R/I } \\
(\mathrm{n}=6)\end{array}$ \\
\hline TOS $(\mu \mathrm{mol} \mathrm{H} 2 \mathrm{O} 2 / \mathrm{L})$ & $108.03 \pm 75.65^{*}$ & $147.97 \pm 32.78^{*}$ & $220.97 \pm 22.85$ & $168.89 \pm 36.93 *$ \\
TAS (mmolTroloxEquiv ) & $13.36 \pm 1.67^{*}$ & $16.27 \pm 3.80^{*}$ & $21.78 \pm 2.47$ & $14.52 \pm 4.37 *$ \\
\hline
\end{tabular}

$\mathrm{P}^{* *}: \mathrm{p}<0.05$ is considered to be significant using Kruskal-Wallis test, $* \mathrm{p}<0.05$ compared to the Group I/R 


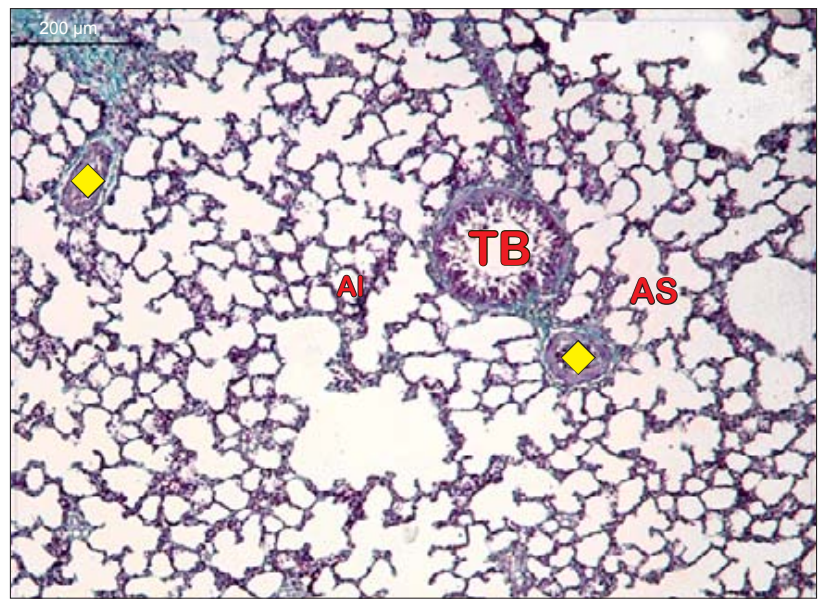

Fig. 1. Lung tissue in Sham Group. TB - Terminal bronchiole, AS - Alveolar sac, Al - Alveol, $\bullet$ - Vessel (Masson-Trichrome X100).

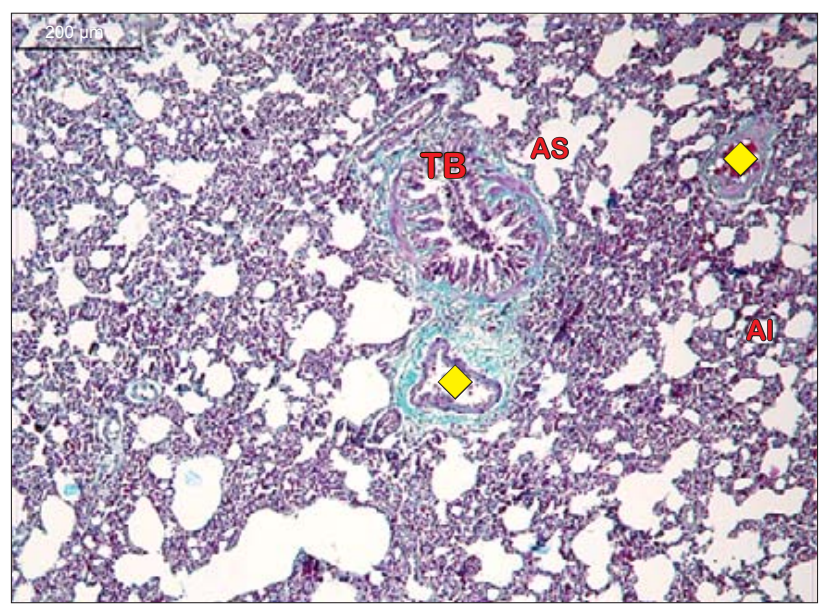

Fig. 3. Lung tissue in Group I/R. TB - Terminal bronchiole, AS - AIveolar sac, Al - Alveol, $\bullet$ - Vessel (Masson-TrichromeX100).

Lot: $903532 \mathrm{~A}$, Invitrogen, USA) in 10 minutes, prior to application of a 1:50 concentration of i-NOS (Cat: sc-651, Lot: H1308, Santa Cruz, USA) for 1 hour. Two PBS rinses preceded secondary antibody (Cat: 859043, Lot: 903532A, Invitrogen, USA) application by 10 minutes. After washing with PBS, slides were exposed to streptavidin peroxidase (Cat: 859043, Lot: 903532A, İnvitrogen, USA) for $10 \mathrm{~min}$. Then AEC (3-Amino-9-EthylCarbazole, Lot: 911667A, Invitrogen, USA) was used as chromogen. Afterwards, the slides were counterstained with Mayer's haematoxylin. Slides were examined with Photo-light microscope (DCM4000 Image Analyze System, DFC280 Plus Camera and QWin Programme, Leica, Weetlar, Germany).

\section{Statistical analyses}

Statistical Package for the Social Sciences (SPSS, Chicago, IL, USA) 17.0 program was used for statistical analysis. KolmogorovSmirnov test was used for the comparisons to determine the distribution of all variable groups. Variations in TOS and TAS levels were assessed by using KruskalWallis test. Bonferroni adjusted

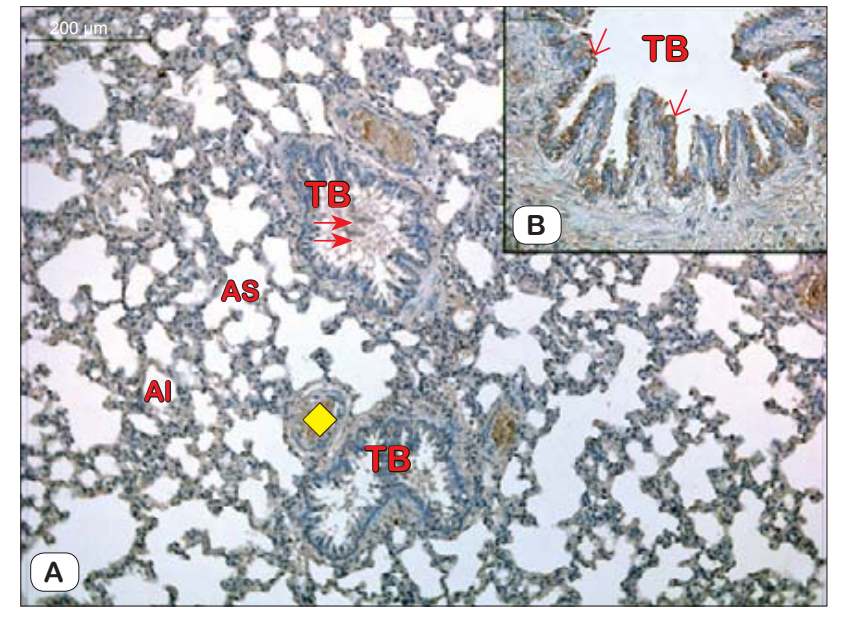

Fig. 2 A,B. INOS expression in lung tissue in Sham Group $(\rightarrow)$. TB - Terminal bronchiole, AS - Alveolar sac, Al - Alveol, $\bullet$ - Vessel, $\rightrightarrows$ - Cellular debris (Immunoperoxidase-Hematoxylin AX100,BX400).

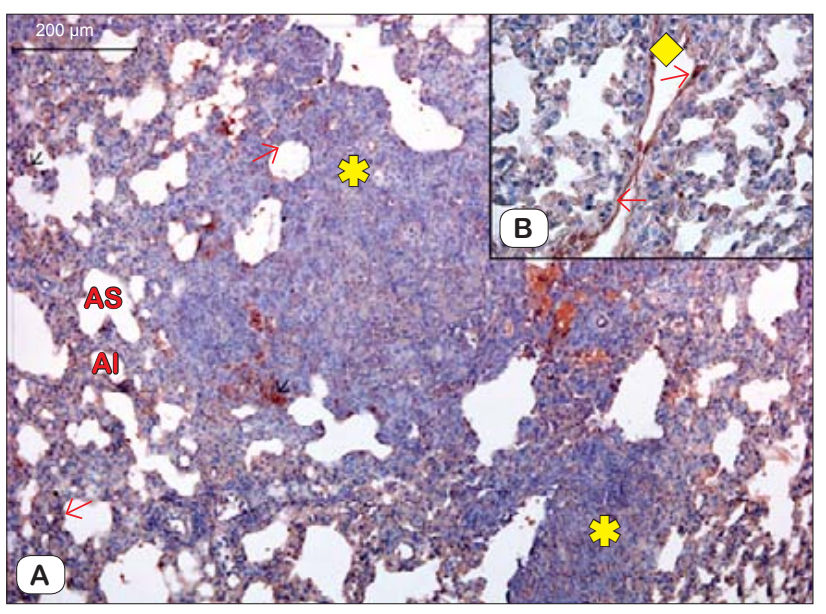

Fig. 4 A,B. INOS expression in lung tissue in Group I/R $(\rightarrow)$. TB Terminal bronchiole, AS - Alveolar sac, Al - Alveol, $\bullet$ - Vessel, $\rightrightarrows$ - Cellular debris, *- Lymphocyte infiltration (ImmunoperoxidaseHematoxylin AX100,BX400).

Mann-Whitney U test was used after significant Kruskal-Wallis to determine which group differs from the other. Results were expressed as mean \pm standard deviation (mean $\pm \mathrm{SD}$ ). Statistical significance was set at a $\mathrm{p}$ value $<0.05$.

\section{Results}

There was a statistically significant difference among the groups when they were compared between themselves by means of TOS levels in lung tissue $(p=0.027)$. TOS levels were significantly higher in Group I/R, when compared with groups S and I $(p=.028, p=0.016$, respectively). TOS levels were found to be significantly lower in group I/R/I, when compared with Group $\mathrm{I} / \mathrm{R}(\mathrm{p}=0.048)$.

A statistically significant difference was found between the groups, when they were compared for TAS levels in lung tissue ( $p$ $=0.019)$. TAS levels were significantly higher in Group I/R, when 
compared with groups $\mathrm{S}, \mathrm{I}$ ( $\mathrm{p}=0.014, \mathrm{p}=0.027$, respectively). TAS levels were found to be significantly lower in Group I/R/I, when compared with Group I/R $(\mathrm{p}=0.032)$. Table 1 shows TOS and TAS parameters in study groups.

The terminal bronchioles, respiratory bronchioles, alveolar ducts and sacs were structurally normal in tissue samples taken from the $\mathrm{S}$ group. Alveolar cells were in normal structure and no cell debris was found in the alveolar lumen. The structure of vascular wall appeared to be normal and no oedema was detected. Immunohistochemical staining demonstrated weak cytoplasmic immunoreactivity for iNOS in vascular structures, apical cytoplasm of bronchial and bronchiolar epithelial cells and particularly in Type-I alveolar cells in this group (Figs 1, 2 A,B).

Diffuse lymphocyte infiltration was detected by immunohistochemical examination of lung tissues in Group I/R. The connective tissue around bronchi, bronchioles and vessel walls was found to be increased. Significant cellular debris was detected in

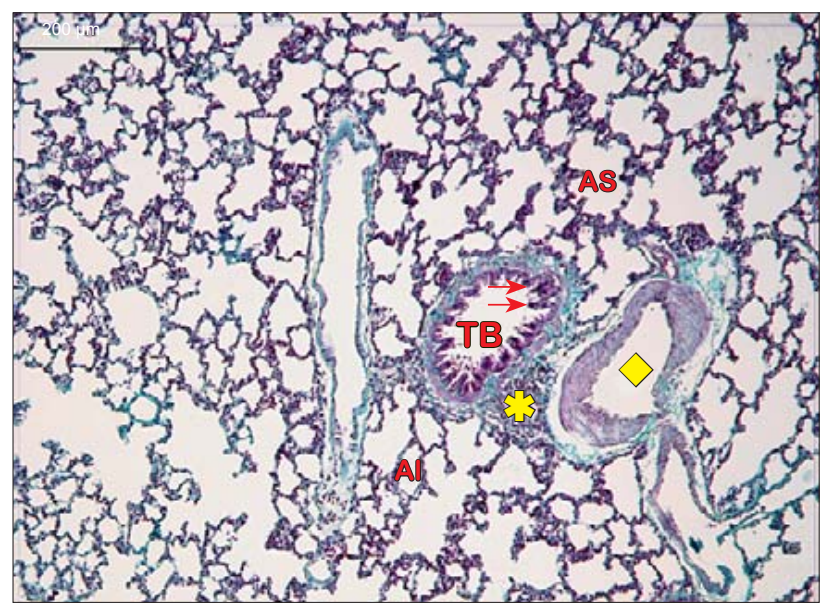

Fig. 5. Lung tissue in Group I. TB - Terminal bronchiole, AS - Alveolar sac, Al-Alveol, $\bullet$ - Vessel, * - Lymphocyte infiltration (MassonTrichromeX100).

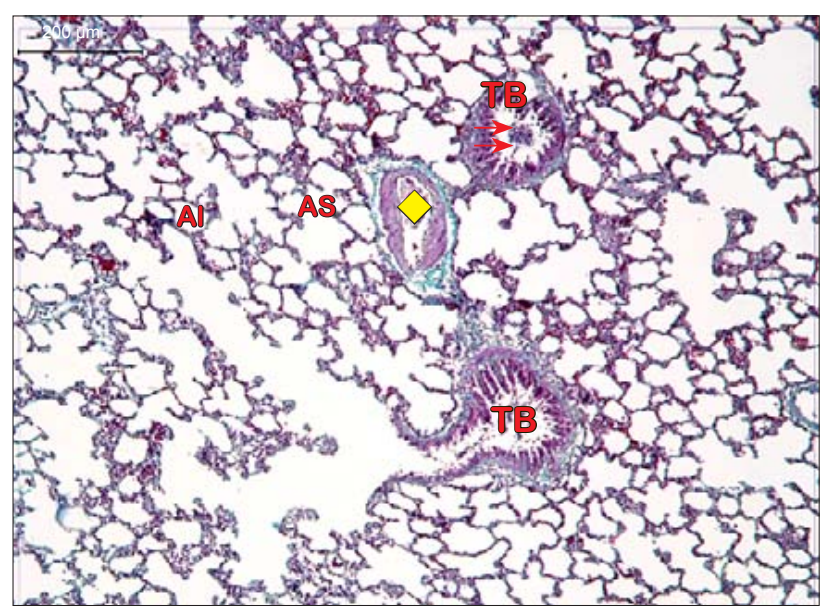

Fig. 7. Lung tissue in Group I/R/I. TB - Terminal bronchiole, AS - Alveolar sac, Al - Alveol, $\bullet$ - Vessel (Masson-TrichromeX100). the lumen of terminal bronchioles. A thickening in the alveolar wall and and an increase in the alveolar connective tissue were noted. A diffuse perivascular oedema was detected in the vascular wall. A significant degeneration in tunica media and degenerative vacuolar structures in the surface of tunica intima facing the lumen were identified. Immunohistochemical examination revealed a significant increase in iNOS expression in this group when compared with the Sham group. The expression was found to be extremely strong in the vascular wall and endothelial cells (Figs 3, 4 A,B).

In Group I, although all lung tissue components were similar to those in S group, some cellular debris was detected in terminal bronchioles. Perivascular oedema and vascular dilatation were found in this group similar to Group I/R. Contrary to Group I/R, no lymphocyte infiltration was detected in Group I. iNOS expression was also detected in all structures mentioned in the $\mathrm{S}$ group, particularly in the connective tissue and it was noted that immunoreactivity was stronger than that of the $\mathrm{S}$ group (Figs 5, $6 \mathrm{~A}, \mathrm{~B}$ ).

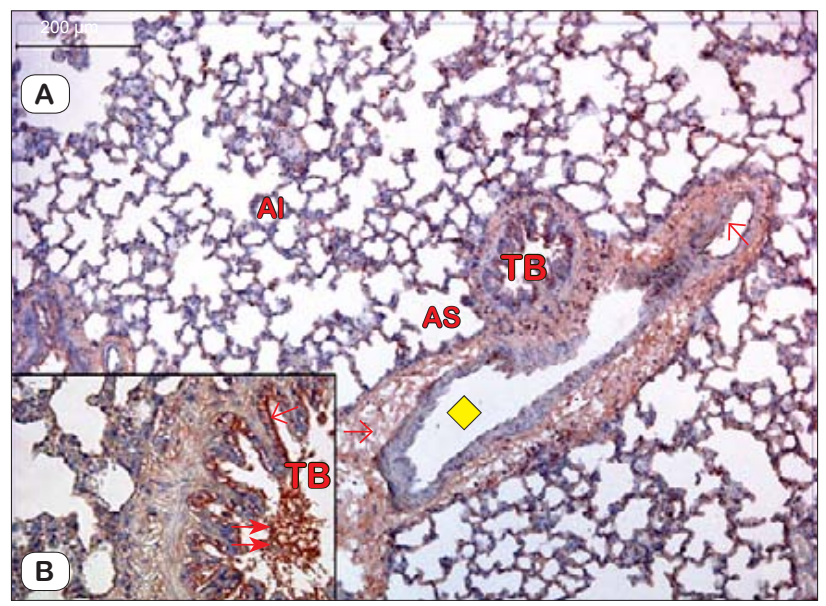

Fig. 6 A,B. INOS expression in lung tissue in Group I $(\rightarrow)$. TB - Terminal bronchiole, AS - Alveolar sac, Al - Alveol, $\bullet-$ Vessel, $\rightrightarrows$ - Cellular debris (Immunoperoxidase-Hematoxylin AX100,BX400).

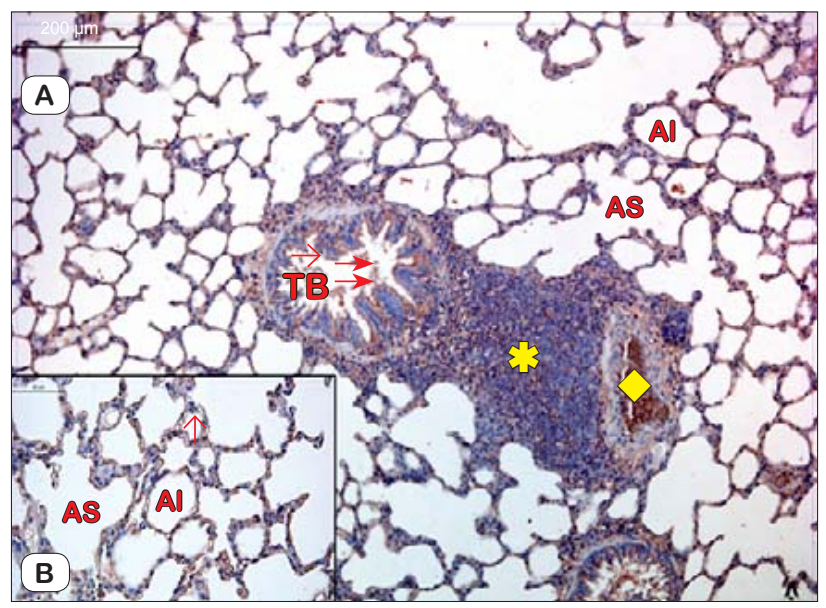

Fig. 8 A,B. INOS expression in lung tissue in Group I/R/I $(\rightarrow)$, TB Terminal bronchiole, AS - Alveolar sac, Al - Alveol, $\bullet$ - Vessel, $\rightrightarrows$ -: Cellular debris, * - BALT (Bronchus associated lymphoid tissue) (Immunoperoxidase-Hematoxylin AX100,BX400). 
Generally, the examination of tissue samples revealed similar findings with the S group, minimal local lymphocyte infiltration was detected in immunohistochemical examination of lung tissues in Group I/R/I. Local cellular debris was observed in some terminal bronchioles and immunohistochemical examination revealed a decreased iNOS expresion compared to Group I/R (Figs 7, 8 A,B).

\section{Discussion}

Remote organ damage caused by I/R of the lower limb targets the lungs, a fact which is of major importance. I/R of the lower limb leads to pulmonary edema by means of pulmonary vasoconstriction, pulmonary hypertension and a rise in alveolar membrane permeability (8).

Temporary aortic cross-clamping is accompanied by I/R injury and a systemic inflammatory response syndrome that can lead to non-cardiogenic pulmonary oedema, or acute respiratory distress syndrome in abdominal aortic surgery $(8,9)$. Various drugs have been reported to reduce I/R/I during aortic cross clamping $(10,11)$.

In the development of lung injury, the excessive generation of reactive oxygen radicals, polymorphonuclear sequestration in pulmonary microvasculature, increased endothelial permeability and interstitial edema have been suggested (12). Restoration of the blood supply to the ischemic tissue results in generation of reactive oxygen species (ROS). Excessive production of ROS causes lipid peroxidation in cell membranes and oxidative damage to DNA and proteins (13). A number of agents, such as $\mathrm{N}$-acetylcystein, calcium dobesilate, aprotinin, erdosteine or magnesium sulfate have been proposed to be useful against lung injury induced by I/R (14-18).

The neuroprotective effect of iloprost, a stable prostacyclin analog, has received attention and has been used in some experimental studies. Iloprost mimics the pharmacodynamic properties of prostacyclin, such as the inhibition of platelet aggregation, vasodilatation and cytoprotection (19). Pretreatment with iloprost decreases pulmonary injury after ischemia $(20,21)$.

In two publications a reversible and dose-related inhibition of the $\mathrm{O}_{2} \bullet-$ production (by neutrophils) was demonstrated in animal models of ischemic myocardium damage in the presence of I (22, $23)$. In addition, I has proven to significantly decrease malondialdehyde (MDA) in animal models of abdominal aortic I/R (24) and induced liver peroxidation (25) and to reduce $\mathrm{H}_{2} \mathrm{O}_{2}$ - induced pathological glaucomatous changes in cultured cells (26).

Iloprost activity in preserving scavenging system has also been reported to positively affect heart transplantation in an animal model when added to cardioplegic solutions (27). Similarly, pretreatment of isolated hearts with iloprost improved myocardial and SOD levels in I/R injury (28).

Therefore, through suppression of nitric oxide expression and subsequent ROS accumulation, Iloprost would directly reduce OS related tissue damage and profibrotic intracellular signalling (4).

Acute ischemia of the lower extremities results in a significant increase in nitric oxide (NO) production from the. NO is a significant determinant of lung injury process induced by lower $\operatorname{limb} \mathrm{I} / \mathrm{R}$. NO can react with other free radicals such as superoxide anion to generate molecules such as peroxynitrite that enhances its cytotoxicity and it is reported to inhibit the aggregation, adhesion and secretory response of polymorphonuclear leukocytes (29). Endothelial cells activate the genes increasing endothelial nitric oxide synthase that catalyses the production of $\mathrm{NO}$ and increased NO levels may contribute to attenuation of I/R injury(30).

In this study, we also used a novel measurement method to evaluate the extent of oxidative stress in rat lungs after skeletal muscle I/R. This provides a useful method for the rapid evaluation of the TAS and TOS, which are valuable parameters in conditions involving oxidative stress. TOS indicates the total oxidative products in tissue. Oxidative products such as ROS, reactive nitrogen species, hydrochloric acid, MDA, and lipid peroxides constitute TOS (6). In our study, TOS levels significantly increased after aortic I/R. We also found that I/R plus I significantly reduced the levels of TOS. TAS levels significantly increased in Group I/R/I. Our findings are consistent with previous papers reporting the antioxidant effects of I on animal models of remote organ injury induced by aortic I/R (20-24). The mechanism of protective effect of I against lung injury can not be explained by its antioxidative effect only, since I/R injury is a complex process. We have hypothesized that it may also have an antioxidative effect on lung injury to some extent. Our findings need to be supported by further studies evaluating different oxidative parameters.

In this study, we aimed to reveal the increasing expression of iNOS in parallel with endothelial injury in lungs using an immunohistochemical methods. Our findings were consistent with the results of biochemical analyses. We observed no immunohistochemical activity in the S group, while a strong staining revealing an increased immunoreactivity was detected in tissue samples taken from Group I/R and a significantly weaker staining was observed in samples in Group I/R/I.

\section{Conclusion}

Immunohistochemical and biochemical findings of this study demonstrate that administration of iloprost may have protective effects against lung injury induced by lower extremity I/R injury and encourage us to investigate this agent in different dosage strategies with alternate administration protocols. Further studies evaluating histological and other biochemical parameters are required to confirm our findings and to elucidate the exact mechanisms of action before clinical use.

\section{References}

1. Avci G, Kadioglu, Sehirli AO et al. Curcumin protects against ischemia/reperfusion injury in rat skeletal muscle. J Surg Res 2012; 172: e39.

2. Beyersdorf F, Unger A, Wildhirt A. Studies of reperfusion injury in skeletal muscle: preserved cellular viability after extended periods of warm ischemia. J Cardiovasc Surg 1991; 32: 664.

3. Eltzschig HK, Collard CD. Vascular ischemia and reperfusion injury. Br Med Bull 2004; 70 (1): 71.

4. Erre GL, Passiu G. Antioxidant effect of Iloprost: current knowledge and therapeutic implications for systemic sclerosis. Reumatismo 2009; 61 (2): 90-97. 
5. Lessiani G, Vazzana N, Cuccurullo $C$ et al. Inflammation, oxidative stress and platelet activation in aspirin treated critical limb ischaemia: Beneficial effects of iloprost. Thromb Haemost 2011; 105: 321-328.

6. Erel O. A new automated colorimetric method for measuring total oxidant status. Clin Biochem 2005; 38: 1103.

7. Erel O. A novel automated direct measurement method for total antioxidant capacity using a new generation, more stable ABTS radical cation. Clin Biochem 2004; 37: 277.

8. Groeneveld AB, Raijmakers PG, Rauwerda JA, Hack CE. The inflammatory response to vascular surgery associated ischemia and reperfusion in man: effect on postoperative pulmonary function. Eur J Vasc Endovasc Surg 1997; 14: 351.

9. Blaisdell FW. The pathophysiology of skeletal muscle ischemia and the reperfusion syndrome: a review. Cardiovasc Surg 2002; 10: 620.

10. Wijnen MH, Roumen RM, Vader HL, Goris RJ. A multi antioxidant supplementation reduces damage from ischaemia reperfusion in patients after lower torso ischaemia. A randomised trial. Eur J Vasc Endovasc Surg 2002; 23 (6): 486-490.

11. Ozyurt H, Ozyurt B, Koca $\mathbf{K}$ et al. Caffeic acid phenethyl ester (CAPE) protects rat skeletal muscle againstischemia-reperfusion-induced oxidative stres. Vascul Pharmacol 2007; 47: 108-112.

12. Welbourn CR, Goldman G, Paterson IS et al. Pathophysiology of ischemia reperfusion injury: central role of the neutrophil. Br J Surg 1991; 78: 651.

13. Oyar EO, Kiris I, Gulmen S et al. The protective effect of adrenomedullin on renal injury, in a model of abdominal aorta cross-clamping. Thorac Cardiovasc Surg 2012; 60: 5 .

14. Sotoudeh A, Takhtfooladi MA, Jahanshahi A et al. Effect of N-acetylcysteine on lung injury induced by skeletal muscle ischemia-reperfusion. Histopathological study in rat model. Acta Cir Bras 2012; 27 (2): 168.

15. Bozkurt AK, Konukoglu D, Ustundag N, Yuceyar L, Mayda AS, Calcium dobesilate ameliorates lung injury following lower limb ischemia/ reperfusion. Drugs Exp Clin Res 2002; 28 (4): 127.

16. Koksal C, Bozkurt AK, Ustundag $\mathbf{N}$ et al. Attenuation of acute lung injury following lower limb ischemia/reperfusion: the pharmacological approach. J Cardiovasc Surg 2006; 47 (4): 445.

17. Sirmali M, Uz E, Sirmali R et al. The effects of erdosteine on lung injury induced by the ischemia-reperfusion of the hind-limbs in rats. $\mathrm{J}$ Surg Res 2008; 145 (2): 303.

18. Kao MC, Jan WC, Tsai PS, Wang TY, Huang CJ. Magnesium sulfate mitigates lung injury induced by bilateral lower limb ischemia-reperfusion in rats. J Surg Res 2011; 171 (1): e97.
19. Fantone JC, Marasco WA, Elgas LJ, Ward PA. Stimulus specificity of prostaglandin inhibition of rabbit polymorphonuclear leukocyte lysosomal enzyme release and superoxide anion production. Am J Pathol 1984; 115 (1): 9-16.

20. Baltalarli A, Ozcan V, Bir F, Aybek H, Sacar M, Onem G, Goksin I, Demir S, Teke Z. Ascorbic acid (vitamin C) and iloprost attenuate the lung injury caused by ischemia/reperfusion of the lower extremities of rats. Ann Vasc Surg 2006; 20 (1): 49-55.

21. Bozok S, Ilhan G, Yilmaz Y et al. Protective effects of hyperbaric oxygen and iloprost on ischemia/reperfusion-induced lung injury in a rabbit model. Eur J Med Res 2012; 17: 14

22. Fantone JC, Kinnes DA. Prostaglandin E1 and prostaglandin I 2 modulation of superoxide production by human neutrophils. Biochem Biophys Res Commun 1983; 113: 506-512.

23. Simpson PJ, Mickelson J, Fantone JC, Gallagher KP, Lucchesi BR. Iloprost inhibits neutrophil function in vitro and in vivo and limits experimental infarct size in canine heart. Circ Res 1987; 60: 666-673.

24. Kiris I, Tekin I, Yilmaz N, Sutcu R, Karahan N, Ocal A. Iloprost downregulates expression of adhesion molecules and reduces renal injury induced by abdominal aortic ischemia-reperfusion. Ann Vasc Surg 2009; 23: 212-223.

25. Bursch W, Taper HS, Somer MP, Meyer S, Putz B, Schulte-Hermann R. Histochemical and biochemical studies on the effect of the prostacyclin derivative Iloprost on CCl4-induced lipid peroxidation in rat liver and its significance for hepatoprotection. Hepatology 1989; 9: 830-838.

26. Yu AL, Fuchshofer R, Kampik A, Welge-Lüssen U. Effects of oxidative stress in trabecular meshwork cells are reduced by prostaglandin analogues. Invest Ophthalmol Vis Sci 2008; 49: 4872-4880.

27. Dedeoglu BD, Aytac E, Suzer O, Balci H, Uzun H, Seymen P et al. Donor heart preservation with Iloprost supplemented St. Thomas Hospital cardioplegic solution in isolated rat hearts. Prostaglandins Leukot Essent Fatty Acids 2008; 78: 415-421.

28. Ferrari R, Cargnoni A, Curello S, Boffa GM, Ceconi C. Effects of Iloprost (ZK 36374) on glutathione status during ischaemia and reperfusion of rabbit isolated hearts. Br J Pharmacol 1989; 98: 678-684.

29. Tassiopoulos A, Carlin RE, GaoY. Role of nitric oxide and tumor necrosis factor on lung injury caused by ischemia/reperfusion of the lower extremities. J Vasc Surg 1997; 26: 647-656.

30. Vural KM, Öz MC. Endothelial adhesivity, pulmonary hemodynamics and nitric oxide synthesis in ischemia reperfusion. Eur J Cardiothorac Surg 2000; 18: 348-352.

Received January 19, 2013. Accepted March 31, 2014. 